\title{
ESTRUCTURA FACTORIAL DE LA ESCALA DE SOPORTE SOCIAL SUBJETIVO: VALIDACIÓN EN UNA MUESTRA DE ESTUDIANTES UNIVERSITARIOS CHILENOS
}

\author{
Manuel Cárdenas Castro ${ }^{1 *}$, Jaime Barrientos Delgado ${ }^{2}$ y Emilio Ricci Alvarado ${ }^{2}$ \\ ${ }^{1}$ Universidad de Valparaíso, ${ }^{2}$ Universidad Católica del Norte
}

Recibido, febrero $21 / 2014$

Concepto de evaluación, octubre 21/2014

Aceptado, diciembre 1/2014
Referencia: Cárdenas Castro, M., Barrientos Delgado, J. \& Ricci Alvarado, E. (2015). Estructura factorial de la escala de soporte social subjetivo: validación en una muestra de estudiantes universitarios chilenos. Acta Colombiana de Psicología, 18(1), 95-101. DOI: 10.14718/ACP.2015.18.1.9

Resumen

El objetivo de este artículo es adaptar y validar la Escala de Apoyo Social Subjetivo (EASS, de Vaux et al., 1986), a la vez que describir su estructura factorial. Esta escala está compuesta por 10 ítems distribuidos en dos dimensiones: apoyo familiar y apoyo de los amigos. Se examinan las propiedades psicométricas de la escala y la dimensionalidad de la misma en una muestra de estudiantes universitarios chilenos $(\mathrm{N}=681)$. Los resultados indican que el EASS constituye un instrumento fiable $(\alpha=.86)$ y válido para la medición del apoyo social subjetivo. Las dimensiones de la EASS obtienen entre sí una correlación significativa y moderada $(\mathrm{r}=.41 ; \mathrm{p}<.001)$. Las cargas factoriales para los ítems en el análisis factorial confirmatorio fluctuaron entre .40 y .93 , mostrando buenos índices de ajuste para el modelo de dos factores de primer orden correlacionados (CFI $=.97$, $\mathrm{TLI}=.93, \mathrm{RFI}=.97, \mathrm{IFI}=.97, \mathrm{NFI}=.96$ y RSMEA $=.08$ ).

Palabras clave: Apoyo social subjetivo, apoyo familiar, apoyo de amistades, propiedades psicométricas

\section{FACTOR STRUCTURE OF THE SUBJECTIVE SOCIAL SUPPORT SCALE: VALIDATION WITH A SAMPLE OF UNIVERSITY STUDENTS IN CHILE}

\begin{abstract}
The purpose of this paper is to adapt and validate the Subjective Social Support Scale (Vaux et al, 1986) and to describe its factor structure. The scale consists of 10 items distributed in two dimensions measured in the original instrument: family support and support from friends. The psychometric properties and dimensionality of the scale are examined in a sample of Chilean college students $(\mathrm{N}=681)$. Results show that the scale is a reliable instrument $(\alpha=.86)$ and valid for measuring subjective social support. Its two dimensions show a moderate and significant correlation $(\mathrm{r}=.41 ; \mathrm{p}<.001)$. The factor loadings of the items in the confirmatory factor analysis varied between .40 and .93 , showing good fit indices for the correlated first order two factor model $(\mathrm{CFI}=.97, \mathrm{TLI}=.93, \mathrm{RFI}=.97, \mathrm{IFI}=.97, \mathrm{NFI}=.96$ and RSMEA $=.08)$.

Key words: Subjective social support, family support, support from friends, psychometric properties
\end{abstract}

* Blanco 1215, Of.101, Valparaíso, Chile. E-mail: manuel.cardenas@uv.cl. Este artículo ha sido financiado por los proyectos FONDECYT 1140890 y FONDECYT 1140887 concedidos por la Comisión Nacional de Investigación Científica y Tecnológica (CONICYT). 


\title{
ESTRUTURA FATORIAL DA ESCALA DE SUPORTE SOCIAL SUBJETIVO: VALIDAÇÂO EM UMA MOSTRA DE ESTUDANTES UNIVERSITÁRIOS CHILENOS
}

\author{
Resumo
}

\begin{abstract}
O objetivo deste artigo é adaptar e validar a Escala de Apoio Social Subjetivo (EASS, de Vaux et al., 1986), e ao mesmo tempo descrever sua estrutura fatorial. Esta escala está composta por 10 itens distribuídos em duas dimensões: apoio familiar e apoio dos amigos. Examinam-se as propriedades psicométricas da escala e a dimensionalidade da mesma em uma mostra de estudantes universitários chilenos $(\mathrm{N}=681)$. Os resultados indicam que o EASS constitui um instrumento fiável $(\alpha=.86)$ e válido para a medição do apoio social subjetivo. As dimensões da EASS obtêm entre si uma correlação significativa e moderada $(\mathrm{r}=.41 ; \mathrm{p}<.001)$. As cargas fatoriais para os itens na análise fatorial confirmatória flutuaram entre .40 e .93 , mostrando bons índices de ajuste para o modelo de dois fatores de primeira ordem correlacionados $(\mathrm{CFI}=.97$, TLI $=.93$, $\mathrm{RFI}=.97, \mathrm{IFI}=.97, \mathrm{NFI}=.96$ e RSMEA $=.08$ ).

Palavras chave: Apoio social subjetivo, apoio familiar, apoio de amizades, propriedades psicométricas
\end{abstract}

\section{INTRODUCCIÓN}

El soporte o apoyo social percibido ha sido descrito como la creencia en la existencia o disponibilidad de personas en las que podemos confiar y con las cuales podemos contar al momento de enfrentar alguna dificultad. Específicamente, la definición de Vaux y colaboradores (1986) alude al grado en que una persona cree que es amada y/o estimada por otros, particularmente su familia y sus amigos. Este constructo se ha asociado con diversos indicadores de salud mental. De este modo, un soporte eficiente se asocia con menores riesgos de contraer enfermedades físicas o problemas de salud mental (Tsouna-Hadjis et al., 2000; Barrón \& Sánchez, 2001; McInnis \& White 2001; Martínez, García \& Maya, 2001), así como con el bienestar subjetivo (Siedlecki et al., 2013; Thomas, 2010; Hombrados-Mendieta et al., 2012; Gallegos-Carillo et al., 2009) y otros indicadores de salud (Reid, Stajduhar \& Chappell, 2010). De modo que la percepción de disponibilidad del apoyo social jugará un rol fundamental en el mantenimiento de la integridad física y psíquica de los individuos.

El soporte social subjetivo supone la valoración, no solo de la cantidad de personas de las que se dispone para acudir en momentos de necesidad, sino también del grado de satisfacción con dichas ayudas. De este modo, se realiza una valoración personal de los apoyos, tanto en lo que refiere al número como a la calidad de estos. Del mismo modo, el concepto alude a la inserción del sujeto dentro de una red de transacción entre el individuo y los otros relevantes que la conforman. Estudios recientes han confirmado la relación entre el soporte social y los niveles de integración social (Lin, 2001). Es por ello que algunos autores (Vaux et al, 1986; Hlebec et al., 2009) dividen el apoyo social en componentes específicos tales como una red social de apoyo, conductas de apoyo y evaluaciones de apoyo. Es la red de apoyo la que permite que dicho soporte pueda ser considerado como estable, así como estable sería el tamaño y la composición de dichas redes. Esta estabilidad de la red sólo se vería alterada en períodos de transición durante el desarrollo de la persona. Las conductas de apoyo se refieren a actos específicos y reconocibles como esfuerzos intencionados para ayudar a alguna persona. Finalmente, las evaluaciones de apoyo corresponden a los elementos más subjetivos de los recursos y comportamientos de apoyo. Serían un indicador primario de la eficacia percibida con respecto a las redes y conductas de apoyo (FernándezLansac \& Crespo, 2011).

Por otra parte, existen estudios que han establecido que el apoyo social mitiga la vivencia de emociones negativas (Crespo \& López, 2007), el estrés (Betrabet, 2009; Lavela \& Ather, 2010), la depresión y la ansiedad (Pearce \& Singer, 2006).

El apoyo social permite obtener los recursos necesarios del ambiente cuando éstos resultan indispensables para los individuos (Pierce et al., 1996), de modo que puedan afrontar apropiadamente las exigencias que les presenta una determinada situación (Stroebe \& Stroebe, 1996). Se distinguen dos fuentes estables de apoyo social. Por un lado, se encuentra la familia, la que a través de sus vínculos armoniosos o conflictivos, puede contribuir a niveles diferenciales de bienestar en los individuos. Del otro lado, se encuentran las amistades, las cuales, sobre todo durante ciertos períodos del desarrollo, constituyen una fuente permanente de refuerzo a la identidad de los individuos. Ambos grupos, familia y amigos, constituyen el soporte afectivo necesario para el logro de objetivos. Se han distinguido tres funciones principales del apoyo social: emocional (intimidad, apego y cuidado), instrumental (prestación de ayuda o asistencia material) e informacional (consejo, guía e información) (Cohen \& Wills, 1985). 
A pesar de lo anterior, se han reportado diferencias importantes entre el soporte social de hombres y mujeres en nuestro país (Arechabala \& Miranda, 2002), lo cual tendría su explicación en la facilidad que tienen las mujeres para profundizar en las relaciones de intimidad y en el mayor número de redes de apoyo que las mujeres reportan (Seligman, 2011). De este modo, resulta esperable que si la escala funciona adecuadamente, los hombres reporten niveles significativamente menores de apoyo social subjetivo que las mujeres.

El objetivo de este artículo fue adaptar y validar la escala de soporte social subjetivo de Vaux y colaboradores (1986), la cual posee una versión abreviada, traducida y adaptada en España por Basabe y sus colaboradores (1993), de modo que pueda ser utilizada para su aplicación con muestras nacionales. Un objetivo adicional fue describir su estructura factorial. En nuestro caso hemos realizado el trabajo de validación en estudiantes universitarios chilenos. Disponer de una medida de apoyo social breve y válida en nuestro entorno permitirá incorporarla a instrumentos más amplios de investigación, dado el acuerdo existente sobre el rol mediador del soporte social entre los eventos estresantes y los resultados de salud y bienestar subjetivo (Schulz et al., 2006), así como por la importancia que por sí misma tiene una medida de apoyo social que posee una valoración de ámbitos diferenciada.

\section{MÉTODO}

\section{Muestra}

La muestra quedó compuesta por 681 participantes, 256 hombres $(37.6 \%)$ y 425 mujeres $(62.4 \%)$, cuyas edades fluctuaron entre los 18 y los 34 años $(\mathrm{M}=20.76$ y $\mathrm{SD}=$ 2.47). Todos los participantes eran estudiantes universitarios. Entre las situaciones o eventos estresantes que habían experimentado se encuentran: la muerte de un familiar cercano o amigo (26.7\%), eventos familiares negativos como despido del trabajo o cambio de ciudad (15.7\%), divorcio o separación de los padres (11.3\%), lesión o enfermedad grave $(7.9 \%)$ y accidentes graves $(3.7 \%)$, entre otros.

\section{Instrumento}

El instrumento consta de dos bloques. En el primero se realiza una serie de preguntas de carácter socio-demográfico que permiten describir la muestra (sexo, edad, nivel socioeconómico). En el segundo bloque se presenta la escala de apoyo social subjetivo.

Escala de Apoyo Social Subjetivo (Vaux et al., 1986; Basabe et al. 1993). La escala consta de 10 ítems que evalúan el soporte familiar y el brindado por los amigos, con opciones de respuesta que van desde 1 ("completamente en desacuerdo") hasta 6 ("completamente de acuerdo"). Las puntuaciones altas indican una alta valoración subjetiva de los niveles de apoyo social.

\section{Procedimiento}

El estudio fue presentado a los sujetos como parte de una investigación acerca de las formas de respuesta ante eventos estresantes y sobre el rol del soporte social al momento de afrontar dichas situaciones. A los participantes se les explicó el objetivo de la investigación y se les garantizaron condiciones de confidencialidad y anonimato; posteriormente entregaron su consentimiento para ser incorporados en la investigación. La participación era voluntaria. Los datos fueron recogidos entre abril y junio de 2013.

Análisis de datos

Todos los análisis fueron realizados utilizando los programas estadísticos SPSS 20.0, AMOS 18 y G*Power 3.1.6. Se llevó a cabo un análisis descriptivo (media, desviación típica, simetría y curtosis) sobre todas las variables. Se efectuó un cálculo del coeficiente de fiabilidad de las escalas (alfa de Cronbach) y de correlaciones de Pearson entre las dimensiones de la EASS. Se realizaron análisis de pruebas $t$ de Student y se calcularon los tamaños del efecto ( $\mathrm{d}$ de Cohen) para las diferencias de medias que resultaron significativas. Se puso a prueba el modelo factorial de dos factores correlacionados de primer orden, por medio de un análisis factorial de tipo confirmatorio.

\section{RESULTADOS}

\section{Estadísticos Descriptivos}

En la tabla 1 se presentan las medias, desviaciones típicas, índices de asimetría y curtosis para cada uno de los ítems, dimensiones y puntuación total de la EASS. Todas las medias se encuentran levemente por encima del punto medio de la escala y con niveles de dispersión relativamente similares.

Un análisis de los ítems nos indica que no se detectan casos atípicos ni extremos en las respuestas a ninguno de ellos. La distribución de la mayoría de los ítems presenta una leve asimetría negativa, lo que indica que la forma de los datos se distribuye agrupando casos en la zona baja de la distribución por debajo de la media. Los niveles de curtosis indican que las respuestas que se acumulan en las colas de la distribución son levemente mayores que en el caso de una curva normal. La distribución de las respuestas al conjunto de la escala muestra asimetría negativa y un apuntamiento de tipo leptocúrtico.

Podemos apreciar que las puntuaciones medias son más bien altas en todos los ítems y que las mujeres puntúan más alto en todos ellos. Estas diferencias entre hombres 
y mujeres son significativas tanto para la escala global $(t(676)=-2,74 ; \mathrm{p}=.011 ;$ IC $95 \%[-0.278,-0.035] ; \mathrm{d}=.19)$, como para la dimensión de apoyo de los amigos $(t(676)=$ $-3,01 ; \mathrm{p}=.003 ;$ IC 95\% [-0.377, -0.079$] ; \mathrm{d}=.24)$. Para la dimensión de apoyo familiar no se detectaron diferencias significativas $(t(676)=-1,16 ; \mathrm{p}=.244)$.

\section{Fiabilidad}

La EASS obtiene un coeficiente de fiabilidad de $\alpha=.86$ para el conjunto de los ítems. No se eliminó ningún ítem de la escala, lo cual mejora la fiabilidad del instrumento. La fiabilidad para las dimensiones fue la siguiente: apoyo

Tabla 1

Cargas factoriales y estadísticos descriptivos *medidas de tendencia central, dispersión y forma de distribución)

\begin{tabular}{|c|c|c|c|c|c|c|}
\hline Ítems & $\begin{array}{l}\text { Media (SD) } \\
\text { global } \\
(\mathrm{n}=681)\end{array}$ & $\begin{array}{c}\text { Media (SD) } \\
\text { hombres } \\
(\mathrm{n}=256)\end{array}$ & $\begin{array}{l}\text { Media (SD) } \\
\text { mujeres } \\
(\mathrm{n}=425)\end{array}$ & Asimetría & Curtosis & $\begin{array}{l}\text { Carga } \\
\text { factorial }\end{array}$ \\
\hline $\begin{array}{l}\text { Mis amigos me } \\
\text { respetan. }\end{array}$ & $5,34(1,03)$ & $5,06(1,16)$ & $5,50(0,91)$ & $-1,929$ & 3,889 & .627 \\
\hline $\begin{array}{l}\text { Mi familia se preocupa } \\
\text { mucho por mí. }\end{array}$ & $5,50(0,98)$ & $5,47(1,00)$ & $5,52(0,97)$ & $-2,218$ & 4,595 & .790 \\
\hline $\begin{array}{l}\text { Mi familia me valora } \\
\text { mucho. }\end{array}$ & $5,47(1,02)$ & $5,44(1,08)$ & $5,49(0,98)$ & $-2,141$ & 4,107 & .900 \\
\hline $\begin{array}{l}\text { Puedo confiar en mis } \\
\text { amigos. }\end{array}$ & $4,95(1,23)$ & $4,89(1,30)$ & $4,99(1,19)$ & $-1,243$ & 1,048 & .897 \\
\hline $\begin{array}{l}\text { Soy muy querido por mi } \\
\text { familia. }\end{array}$ & $5,50(0,97)$ & $5,44(1,07)$ & $5,54(0,88)$ & $-2,228$ & 4,806 & .652 \\
\hline $\begin{array}{l}\text { Los miembros de mi } \\
\text { familia confían en mí. }\end{array}$ & $5,38(1,05)$ & $5,34(1,09)$ & $5,41(1,03)$ & -2.032 & 4,039 & .407 \\
\hline $\begin{array}{l}\text { Tengo un fuerte lazo } \\
\text { afectivo con mis } \\
\text { amigos. }\end{array}$ & $5,09(1,16)$ & $4,99(1,14)$ & $5,15(1,17)$ & $-1,390$ & 1,535 & .728 \\
\hline $\begin{array}{l}\text { Mis amigos y yo somos } \\
\text { realmente importantes } \\
\text { los unos para los otros. }\end{array}$ & $4,88(1,22)$ & $4,75(1,72)$ & $4,96(1,24)$ & $-1,536$ & 0.932 & .889 \\
\hline $\begin{array}{l}\text { Me siento integrado en } \\
\text { un grupo de personas. }\end{array}$ & $5,15(1,21)$ & $5,00(1,29)$ & $5,24(1,15)$ & $-1,611$ & 2,130 & .931 \\
\hline $\begin{array}{l}\text { No me siento cercano } \\
\text { a los miembros de mi } \\
\text { familia.* }\end{array}$ & $4,82(1,76)$ & $4,72(1,76)$ & $4,89(1,76)$ & $-1,181$ & $-0,198$ & .905 \\
\hline Escala completa & $5,20(0,78)$ & $5,11(0,83)$ & $5,26(0,74)$ & $-1,415$ & 2,111 & $\alpha=.86$ \\
\hline $\begin{array}{l}\text { Dimensión } 1 \text { (soporte } \\
\text { familiar) }\end{array}$ & $5,33(0,92)$ & $5,28(0,94)$ & $5,36(0,90)$ & $-1,616$ & 2,202 & $\alpha=.83$ \\
\hline $\begin{array}{l}\text { Dimensión } 2 \text { (soporte } \\
\text { amigos) }\end{array}$ & $5,08(0,96)$ & 4,93 $(0,99)$ & $5,16(0,93)$ & $-1,334$ & 1,660 & $\alpha=.88$ \\
\hline
\end{tabular}

(*) Es el único ítem con puntuación inversa. Para nuestro caso ya ha sido invertido, por lo que puntuaciones altas indican mayor cercanía con la familia. 
familiar $\alpha=.83$ y apoyo de amigos $\alpha=.88$. Las dimensiones de la escala se correlacionan de forma positiva y significativa $(r=.41 ; \mathrm{p}<.001)$.

\section{Análisis Factorial Confirmatorio}

Se ha puesto a prueba un modelo de dos factores de primer orden correlacionados entre sí, en el cual cada factor queda compuesto por cinco ítems. Los parámetros del modelo han sido estimados mediante el método de máxima verosimilitud. Se utilizó una serie de índices para estimar el ajuste del modelo, a saber: Chi cuadrado $\left(\mathrm{X}^{2}\right)$, índice de ajuste comparativo (CFI), índice de Tucker-Lewis (TLI), índice de ajuste normalizado (NFI), índice de ajuste relativo (RFI), índice de ajuste incremental (IFI) y raíz cuadrada del error de aproximación (RMSEA). Para el caso de Chicuadrado se espera que no resulte significativo (aunque este indicador no debería utilizarse para rechazar un modelo debido a su sensibilidad frente al tamaño de la muestra), los índices TLI, IFI, RFI NFI y CFI deberían situarse lo más cercanos a 1 , aunque se espera que siempre superen el valor de .90 (Bentler \& Dudgeon, 1996). El valor de RSMEA debe situarse bajo el valor crítico de .08 (Browne \& Cudeck, 1993).

Los datos obtenidos se ajustan bien al modelo teórico, ya que si bien el valor de $\left(\mathrm{X}_{(34)}^{2}=186.63 ; \mathrm{p}<.001\right)$ resulta significativo (se esperaría que no lo fuera, ya que se contrasta la hipótesis nula de ajuste perfecto entre los datos empíricos y teóricos), el valor del índice CFI es de .97, el de TLI de .94, el RFI de .93, el IFI de .97 y el NFI de .96, lo que indica claramente el buen ajuste de los datos. Finalmente, el valor de RMSEA fue .08 (IC 95\% .070, .093), lo cual pone de presente un buen ajuste general del modelo. Las cargas factoriales de todos los ítems se situaron dentro de un rango de .40 hasta .93 (véase Tabla 1).

\section{DISCUSIÓN}

Los resultados del estudio permiten confirmar que la EASS constituye un instrumento fiable y válido para medir los niveles de apoyo social, toda vez que confirma que las relaciones familiares y de amistad resultan sumamente importantes a la hora de afrontar las experiencias estresantes de la vida.

Del mismo modo, hemos verificado la dimensionalidad de dicha escala, la cual se muestra como un instrumento apropiado para recoger las diferentes valoraciones sobre cada grupo, familia y amigos, en cuanto al tipo de apoyo que cada cual brinda. Ambos grupos constituirían una importante fuente de apoyo instrumental y emocional, y facilitan en un alto grado la integración social.

Asimismo, hemos podido apreciar que los puntajes de la dimensión amigos son levemente menores a los obtenidos en la dimensión de apoyo familiar. Lo anterior podría explicarse porque en Chile la familia extendida, en la que se convive con parientes, sigue siendo una realidad frecuente y tiene, al menos durante la mayor parte de la vida, una presencia constante (Muñoz y Reyes, 1997, citado en Arechabala y Miranda, 2002). En cualquier caso, las puntuaciones de ambas dimensiones son elevadas.

Del mismo modo, hemos constatado diferencias entre hombres y mujeres en la escala total y en la dimensión amigos, siendo las puntuaciones de las mujeres más altas que las de los hombres. Esto es congruente con el hecho de que los grupos de amistad suelen ser menos estables que la familia, así como con la existencia de diferencias de género (Belle, 1989; Kessler et al, 1985). Las amistades de los hombres siguen basándose en las actividades compartidas, las cuales disminuyen con el pasar de los años. Del mismo modo, la intimidad de los hombres suele limitarse a la pareja y la familia, mientras que las mujeres cuentan con una red de apoyo emocional mayor que la de los hombres y suelen, debido a factores culturales, establecer relaciones más intensas y de mayor intimidad. Algunos estudios informan de redes de apoyo más amplias en las mujeres (Scott \& Wenberg, 1996), siendo estas mismas capaces de brindar apoyo para otras personas en mayor medida que los hombres (al menos de carácter emocional e informacional). Si bien esto podría resultar cierto, los dos grupos muestran puntuaciones altas en la escala de apoyo social subjetivo.

Los resultados obtenidos nos permiten concluir que la escala posee buenas propiedades psicométricas y resulta por ello un instrumento que mide adecuadamente el apoyo social subjetivo. Este instrumento diferencia claramente dos dimensiones, familia y amigos, que corresponden a un modelo subyacente de dos factores correlacionados entre sí. Esta escala nos permitirá medir de forma simple y confiable la percepción de apoyo de los participantes en futuras investigaciones, toda vez que permite distinguir apropiadamente las fuentes de dicho apoyo.

No obstante lo anterior, este estudio posee una serie de limitaciones que deben ser corregidas en futuros estudios. La principal de estas limitaciones se refiere al tipo de muestreo usado, no probabilístico, debido a que la muestra tiende a ser homogénea y a compartir algunas características como la edad y nivel educativo, las que podrían concebirse como variables relevantes a la hora de especificar los niveles de soporte social de una persona. 


\section{REFERENCIAS}

Arechabala, M.C. \& Miranda, C. (2002). Validación de una escala de apoyo social percibido en un grupo de adultos mayores adscritos a un programa de hipertensión de la región Metropolitana. Ciencia y Enfermería, 8, 1, 49-55. doi: 10.4067/S0717-95532002000100007

Barrón, A. \& Sánchez, E. (2001). Estructura social, apoyo social y salud mental. Psicothema, 13(1), 17-23.

Basabe, N., Valdemosa, M. \& Páez, D. (1993). Memoria afectiva, salud, formas de afrontamiento y soporte social. En: D. Páez (Ed.). Salud, expresión y represión social de las emociones. Valencia: Promolibro.

Belle, D. (1989). Gender differences in children's social networks and support. In D. Belle (Ed.), Children's social networks and social supports (p 173-188). New York: Wiley.

Bentler, P. M., \& Dudgeon, P. (1996). Covariance structure analysis: Statistical practice, theory, directions. Annual Review of Psychology, 47, 563-592. doi: 10.1146/annurev. psych.47.1.563

Betrabet, G. (2009). Restorative home environments for family caregivers. Journal of Aging Studies, 23(3), 197-204. doi: 10.1016/j.jaging.2007.11.005

Browne, M. W., \& Cudeck, R. (1993). Alternative ways of assessing model fit. En: K. A. Bollen \& J. S. Long (Eds.), Testing structural equation models (pp. 136-162). Newsbury Park, CA: Sage.

Cohen, S., \& Wills, T.A. (1985). Stress, social support and the buffering hypothesis. Psychological Bulletin, 98, 310-357. doi: 10.1037/0033-2909.98.2.310

Crespo, M. \& López, J. (2007). El apoyo a los cuidadores de familiares mayores dependientes en el hogar: desarrollo del programa "Cómo mantener su bienestar". Madrid: Ministerio de Trabajo y Asuntos Sociales, Secretaría de Estado de Servicios Sociales, Familias y Discapacidad, Instituto de Mayores y Servicios Sociales (IMSERSO).

Fernández-Lansac, V. \& Crespo, M. (2011). Resiliencia, personalidad resistente y crecimiento en cuidadores de personas con demencia en el entorno familiar: una revisión. Clínica y Salud, 22(1), 21-40.

Gallegos-Carrillo, K., Mudgal, J., Sánchez-García, S., Wagner, F. A., Gallo, J. J., Salmerón, J., et al. (2009). Social networks and health-related quality of life: A population based study among older adults. Salud Pública de México, 51(1), 6-13. doi: 10.1590/S0036-36342009000100004

Hlebec, V., Mrzel, M., \& Kogovšek, T. (2009). Social Support Network and Received Support at Stressful Events. Metodološki zvezki, 6(2), 155-171.
Hombrados-Mendieta, I., García-Martín, M.A., \& GómezJacinto, L. (2012). The Relationship Between Social Support, Loneliness, and Subjective Well-Being in a Spanish Sample from a Multidimensional Perspective. Social Indicators Research, 1-22. doi: 10.1007/s11205012-0187-5

Kessler, R. C., McLeod, J. D., \& Wethington, E. (1985). The costs of caring: A perspective on the relationship between sex and psychological distress. In I. G. Sarason \& B. R. Sarason (Eds.), Social Support: Theory, research and applications. Dordrecht, Netherlands: Martinus Nijhoff.

Lavela, S. L. \& Ather, N. (2010). Psychological health in older adult spousal caregivers of older adults. Chronic Illness, 6(1), 67-80. doi: 10.1177/1742395309356943

Lin, N. (2001). Social capital: a theory of social structure and action. Cambrigde, UK: Cambridge University Press.

Martínez, F.M., García, M. \& Maya, I. (2001). El efecto amortiguador del apoyo social sobre la depresión en un colectivo de inmigrantes. Psicothema, 13(4), 605-610.

McInnis, G.J. \& White, J.H. (2001). A phenomenological exploration of loneliness in the older adult. Archives of Psychiatric Nursing, 15(3), 128-139.

Pearce, M. J. \& Singer, J. L. (2006). Religious coping among caregivers of terminally ill cancer patients. Journal of Health Psychology, 11(5), 743-759. doi:10.1177/1359105306066629

Pierce, G. R., Sarason, I. G. \& Sarason, B. R. (1996). Coping and social support. En: M. Zeidner \& N. Endler (Eds.), Handbook of coping: Theory, research, applications (pp. 434-451). Nueva York: Wiley.

Reid, R. C., Stajduhar, K. I. \& Chappell, N. L. (2010). The impact of work interferences on family caregiver outcomes. Journal of Applied Gerontology, 29(3), 267-289. doi: 10.1177/0733464809339591

Schulz, A.J., Israel, B.A., Zenk, S.N., Parker, E.A., Lichtenstein, R., Shellman-Weir, S., \& Klem, L. (2006). Psychosocial stress and social support as mediators of relationships between income, length of residence and depressive symptoms among African American women on Detroit's eastside. Social Science \& Medicine, 62(2), 510-522. doi. org/10.1016/j.socscimed.2005.06.028

Scott, A. y Wenger G. (1996). Género y redes de apoyo social en la vejez. En S. Arber y J Ginn (Comps). Relación entre género y envejecimiento: Enfoque sociológico. Madrid: Narcea S.A. Editores.

Seligman, M. (2011). La auténtica felicidad. Barcelona: Ediciones Zeta.

Siedlecki, K.L., Salthouse, T.A., Oishi, S., \& Jeswani, S. (2013). The Relationship Between Social Support and Subjective Well-Being Across Age. Social Indicators Research, 112(2), doi: 10.1007/s11205-013-0361-4 
Stroebe, W. \& Stroebe, M. (1996). The social psychology of social support. En: T. Higgins \& W. Kruglanski, Social psychology: Handbook of basic principles (pp. 597-622). Nueva York: The Guilford Press.

Tsouna-Hadjis, E., Vemmos, K.N., Zakopoulos, N. \& Stamatelopoulos, S. (2000). First-stroke recovery process: the role of family social support. Archives of Physical
Medicine and Rehabilitation, 81(7), 881-887. doi:10.1053/ apmr.2000.4435

Vaux, A., Phillips, J., Holly, L., Thomson, B., Williams, D., \& Stewart, D. (1986). The social support appraisals (SS-A) scale: Studies of reliability and validity. American Journal of Community Psychology, 14, 195-219. doi: 10.1007/ BF00911821 